\title{
INTERTROCHANTERIC FRACTURES; FREQUENCY OF LAG SCREW CUT OUT FIXED WITH DYNAMIC HIP SCREW
}

1. MBBS, FCPS (Orthopaedics) Assistant Professor,

Orthopaedics \& Traumatology Unit"B"

Medical Teaching Institution (MTI)

Lady Reading Hospital (LRH) Peshawar.

2. MBBS, FCPS (Orthopaedics), MRCS, AO fellow (spine)

Senior Registrar, Orthopaedic Unit Saidu Medical College Swat.

3. Associate Professor

Incharge Orthpaedics B Unit

Lady Reading Hospital Peshawar.

Correspondence Address:

Dr. Faaiz Ali Shah

Assistant Professor,

Orthopaedics \& Traumatology Unit "B"

Medical Teaching Institution (MTI)

Lady Reading Hospital (LRH)

Peshawar.

Article received on:

06/02/2017

Accepted for publication:

$15 / 09 / 2017$

Received after proof reading:

$03 / 11 / 2017$

\section{Faaiz Ali Shah', Waqar Alam², Mian Amjad Ali ${ }^{3}$}

ABSTRACT... Objectives: To determine the frequency of lag screw cut out in intertrochanteric fractures fixed with dynamic hip screw and to identify the possible contributing factors for screw cut out. Study Design: Descriptive case series. Place and Duration of the Study: Orthopaedic \& Traumatology Unit"A" Lady. Reading Hospital Peshawar from January 2014 to January 2017. Material and Methods: Patients of either gender or age with intertrochanteric fractures fulfilling the inclusion and exclusion criteria were fixed with dynamic hip screw (DHS). Post operatively fracture reduction, position of screw in head of femur and tip apex distance were calculated on anterio posterior and lateral radiographs. All the patients were reviewed fortnightly for two month and then monthly for six months for lag screw cut out on radiographs. Results: Total 110 patients mean age 72 (range 22 to 98 years) years were fixed with dynamic hip screw. Lag screw cut out was noted in $12(10.9 \%)$ patients with $4(33.3 \%)$ male and $8(66.6 \%)$ female patients. The mean age was 62 years. Right side was involved in two (16.6\%) patients while left in ten (83.3\%) patients. Radiographically fracture reduction was poor in most $(50 \%, \mathrm{n}=6))$ patients. The mean tip apex distance (TAD) was $32 \mathrm{~mm}$ (range 24 to $40 \mathrm{~mm}$ ). Majority $(75 \%, n=9)$ of cut out screw were in superior portion of the head of femur. Four (33.3\%) patients had screw cut out at $8^{\text {th }}$ week postoperatively while eight $(66.6 \%)$ patients had screw cut out at $12^{\text {th }}$ week. Conclusion: Lag screw cut out wasthe most common mechanical complication after fixation of intertrochanteric fractures and all of the cut out patients were elderly with inadequately reduced fracture, superiorly placed lag screw and longer tip apex distance.

Key words: Intertrochanteric fracture, dynamic hip screw, tip apex distance.

Article Citation: Shah FA, Alam W, Ali MA. Intertrochanteric fractures; Frequency of lag screw cut out fixed with dynamic hip screw. Professional Med J 2017;24(11):17401744. DOI:10.17957/TPMJ/17.3867

\section{INTRODUCTION}

Hip fractures commonly occur in old age with osteoporosis and intertrochanteric fracture is the common one. ${ }^{1}$ Approximally 296,000 hip fractures are reported annually in United States and intertrochanteric fractures constitute half of these fractures. ${ }^{2}$ Various implants like dynamic hip screw (DHS), angular blade plate and intramedullary nails are used to treat intertrochanteric fractures. ${ }^{3}$ Dynamic hip screw(DHS) is an extramedullary implant which is relatively simple, safe and favored device to fix these fractures. ${ }^{4,5}$ Although dynamic hip screw is a time tested device, lag screw cut out is the most common mechanical failure of this implant reported in $8 \%$ of cases in the literature. ${ }^{6}$ In 1995 Baumgaertner ${ }^{7}$ in his classical landmark study showed that although multiple risk factors like old age and osteoporosis, unstable fracture type, poor fracture reduction or fixation and use of 150 degree angle implants were responsible for screw cut out but the most important and the gold standard predictor of lag screw cut out he identified was the tip apex distance (TAD)which is the sum of distances from the tip of the lag screw to the apex of femoral head measured on anterio-posterior view $\left(X_{a p}\right)$ and lateral view ( $X$ (at) after magnification is corrected by using the true diameter $\left(D_{\text {true }}\right)$ of the shaft of lag screw as a reference. (Figure-1) Baumgaertner ${ }^{7}$ further noted that there were no screw cut-out in patients with a tip apex distance (TAD) of less than $25 \mathrm{~mm}$ but $27 \%$ of his patients had cut out of lag screw because they had a TAD of more than $30 \mathrm{~mm}$. Furthermore his results supported the central placement of lag screw to obtain tip apex distance of less than $25 \mathrm{~mm}$. Subsequent studies. ${ }^{8,9,10}$ supported 
his recommendations. Screw cut out in patients with a hip fracture require another surgery which might not be tolerated by these elderly patients because of the existing comorbidities associated with advancing age. The purpose of this study was to determine the frequency of lag screw cut out in patients with intertrochanteric fractures fixed with dynamic hip screw. This study will also identify the possible factors that may contribute to screw cut out. This will be the first study on this topic in our institution.

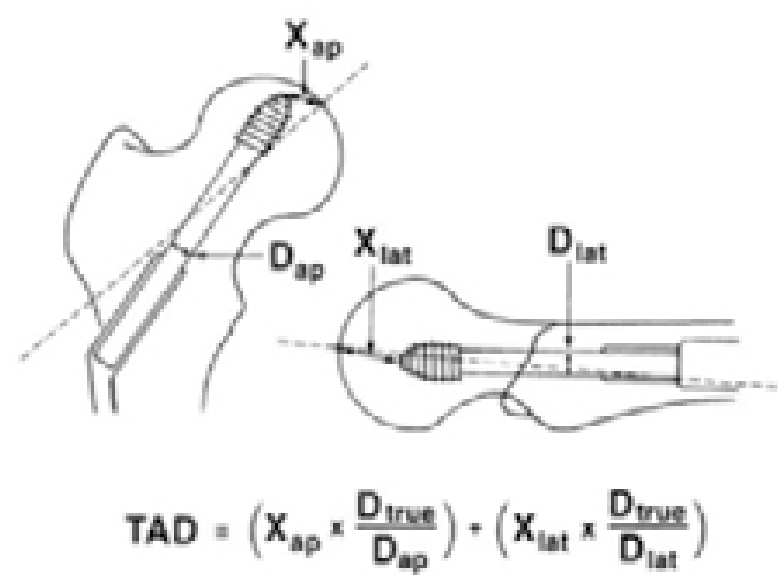

Figure-1. Technique for the calculation of tip apex distance (TAD)

\section{MATERIAL AND METHODS}

This descriptive case study was conducted in Orthopaedic\& Traumatology Unit "A" Lady Reading Hospital Peshawar from January 2014 to January 2017.Patients of either gender and age with intertrochanteric fractures which were fixed with dynamic hip screw (DHS) were included in the study. Patients with revision hip surgery, pathological fractures, polytrauma patients with multiple fractures, patients with in hospital mortality or those died within six months post operatively, those unable to complete follow up for at least first six months post operatively and those with inadequate or missing radiographs were excluded from the study. The research protocols were approved by the hospital Ehical Review Board. Informed written consent from the participants were taken. In the included subjects pre operative complete history and physical examination was done. X-ray AP \& Lateral view of the affected hip was taken and fracture was classified according to Orthopedic
Trauma Association (AO/OTA) fracture classification. ${ }^{11}$ All the fractures were fixed with DHS (135degrees) under image intensifier with optimum surgical skills taking into consideration the correct position of lag screw as advocated by Baumgaertner. ${ }^{7}$ Immediatepost operative check X-ray of the hip AP \& Lateral view were done in all cases. The fracture reduction was labelled as Good when the fracture alignment was normal or slight valgus on AP X-ray, not more than 20 degree angulation on lateral $x$-ray and less than $4 \mathrm{~mm}$ displacement of any fracture fragment. The reduction was Acceptable when either alignment or displacement was of good quality but not the both and Poor when both were not present. ${ }^{7}$

Tip apex distance (TAD) was calculated on AP \& Lateral X-ray according to Baumgaertner's formula. $^{7}$ (Figure-1) The position of the lag screw in femoral head was located in one of the nine zones superior, central and inferior on AP x-ray and anterior central and posterior on lateral $\mathrm{X}$-ray. ${ }^{12,13}$ All post operative patients received the uniform standard postoperative protocol of protected weight bearing with a walking stick for two months. All the patients were regularly followed fortnightly for first two months and then monthly for six months. In each visit lag screw position was radiologically assessed for cut out (protrusion of lag screw from femoral head by more than $1 \mathrm{~mm}$ ). 14 Data collected was entered into SPSS (version 16) for statistical analyses.

\section{RESULTS}

A total of 110 patients mean age 72 years (range 22 to 98 ) were included in the study. Male patients were $64(58.1 \%)$ while female patients were 46(41.8\%). Right intertrochentric fracture was present in $78(70.9 \%)$ while left in $32(29 \%)$ patients. The aetiology of fracture was fall in $80(72.7 \%)$ and road traffic accidents in $30(27.2 \%)$ patients. The fracture type was AO type 31A1 in 45(40.9\%) and $A O 31 A 2$ in $65(59 \%)$ patients. There was no screw cut out in $90(81.8 \%)$ patients with mean TAD 22mm (range 18 to $38 \mathrm{~mm}$ ). Lag screw cut out was found in $12(10.9 \%)$ patients with $4(33.3 \%)$ male and $8(66.6 \%)$ female patients. The mean age was 62 years. AO type $31 \mathrm{~A} 1$ was present in 4(33.3) patients while AO type 31A2 was present 
in $8(66.6 \%)$ patients. Radiographically fracture reduction was good in $2(16.6 \%)$, acceptable in $4(33.3 \%)$ and poor in $6(50 \%)$ patients. The mean tip apex distance (TAD) was $32 \mathrm{~mm}$ (range 24 to $40 \mathrm{~mm}$ ) in screw cut out group. The location of lag screw in femoral head according to the zones are shown in Figure-2. Majority $(75 \%, n=9)$ of cut out screw were in superior portion of the head of femur. Four (33.3\%) of our patients had screw cut out at $8^{\text {th }}$ week postoperatively while eight $(66.6 \%)$ patients had screw cut out at $12^{\text {th }}$ week. Right side was involved in two (16.6\%) patients while left in ten $(83.3 \%)$ patients. No breakage of implant was noted. Revision surgery was performed in $8(66.6 \%)$ patients, implant removal followed by skeletal traction in $2(16.6 \%)$ while $2(16.6 \%)$ patients refused revision surgery.

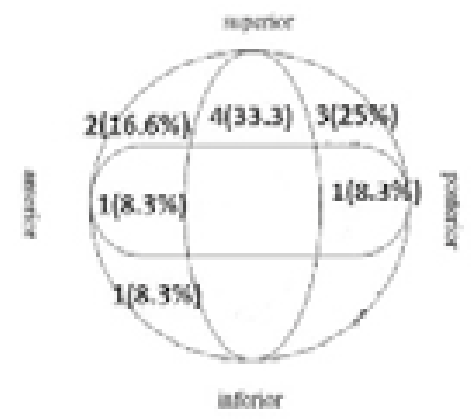

Figure-2. Distribution of lag screw cut out in femoral head according to Cleveland's zones.

\section{DISCUSSION}

The lag screw cut out rate in literature varies from $1.6 \%$ to $36.4 \%$ (table 1 ) while in our study it was $10.9 \%$ comparable to previous studies. With regard to the screw position in femoral head, majority of screw cut out in our study were in superior and posterio superior position (33.3\% and $25 \%$ respectively) according to the Cleveland's ${ }^{12}$ nine zones of femoral head while no screw cut was noted in central or middle/middle,central inferior and posterior inferior position. Similar to our study Hsueh ${ }^{16}$ reported highest lag screw cut out rates $(36.8 \%$ and $33.3 \%)$ in superior and posterior location while lowest cut out in middle/middle or central position (2.1\%) and they proposed the central or middle/middle position as the best position for lag screw placement in hip fracture fixation. Other studies ${ }^{7,14,19}$ support the same findings.
The mean tip apex distance (TAD) was $32 \mathrm{~mm}$ (range 24 to $40 \mathrm{~mm}$ ) in screw cut out group in our study. Andruszkowet al ${ }^{18}$ evaluated 188 patients of DHS and documented a higher incidence of cut out rates with anterior placement of lag screw and twenty four times increased risk of lag screw cut out when TAD exceeded $25 \mathrm{~mm}$. Other studies $^{9,10,20}$ had the same observation that a TAD of greater than $25 \mathrm{~mm}$ is a strong risk factor for lag screw cut out. Hsueh ${ }^{16}$ reported 11 cases of lag screw cut out with TAD $<25 \mathrm{~mm}$, two cases of cu out with $T A D<20 \mathrm{~mm}$ and no cut out in patients with TAD $<15 \mathrm{~mm}$. He suggested that to avoid lag screw cut out TAD should be less than $15 \mathrm{~mm}$.he also noted that screw position had a relation with TAD i.e the more peripheral(posterior or superior) the position of the screw the more longer the TAD. Bruijn ${ }^{19}$ noted the threshold of TAD $19.9 \mathrm{~mm}$ in his study. In our study we also observed that majority $(66.6 \%)$ of our patients in the cut out group had unstable fractures (AO type 31A2) and reduction was poor (50\%) in most of them. This has been confirmed by other studies. ${ }^{15,16}$

An interesting findings of our study was that majority $(83.3 \%, n=10)$ of cut out patients had involvement of left sided hip. This can be explained by a theory proposed by Mohan ${ }^{23}$ that left intertrochanteric fractures have a tendency for displacement of their proximal fragment because of the torque created when the lag screw is rotated clockwise for insertion into the head of femur and as a result fracture is fixed in a poorly reduced position and with ambulation further torque is applied causing gradual cut out of lag screw from femur head. On the other hand this torque effect of lag screw rotation causes compression of the right sided intertrochanteric fractures and further stabilizes the fracture and reducing risk of screw cut out. This theory is not only supported by Goh ${ }^{21}$ but he also proposed provisional kirschner wire fixation of the main fracture fragments in order to reduce the excessive torque while inserting the lag screw in left intertrochanteric fractures.

Our study had some limitations or weaknesses. TAD was measured by the principal author alone on radiographs and errors in uniform measurements was a possibility. This error could be minimize by 
using digital system such as Picture Archiving and Communication System (PACS) ${ }^{22}$ which is more accurate and reproducible but unfortunately which was not available to us. Furthermore bone mineral density (BMD) of our patients could not be evaluated and no pre surgery protocols with regard to TAD and screw position was set. We recommend that larger number of patients should be evaluated to confirm our observations.

\begin{tabular}{|c|l|c|c|}
\hline $\begin{array}{c}\text { S. } \\
\text { No }\end{array}$ & \multicolumn{1}{|c|}{ Study Name } & Year & $\begin{array}{c}\text { Dhs Lag Screw } \\
\text { Cut Out }\end{array}$ \\
\hline 1 & ${\text { Guven et al. }{ }^{15}}^{16}$ & 2010 & $5.8-7.1 \%$ \\
\hline 2 & Hsueh $^{16}$ & 2010 & $6.8 \%$ \\
\hline 3 & Agni $^{17}$ & 2012 & $1.6 \%$ \\
\hline 4 & Andruszkow et al. $^{18}$ & 2012 & $3.2 \%$ \\
\hline 5 & Sedighi $^{6}$ & 2012 & $8 \%$ \\
\hline 6 & Bruijn $^{19}$ & 2012 & $19 \%$ \\
\hline 7 & Imadudin $^{20}$ & 2014 & $2-36.4 \%$ \\
\hline 8 & Goh $^{21}$ & 2016 & $6 \%$ \\
\hline 9 & Our study & 2017 & $10.9 \%$ \\
\hline Table-I. Frequency of dynamic hip lag screw (DHS) \\
\hline \multicolumn{2}{|c}{ cut out in different studies. } \\
\hline
\end{tabular}

\section{CONCLUSION}

Lag screw cut out was the most common mechanical complication after fixation of intertrochanteric fractures and all of the cut out patients were elderly with inadequately reduced fracture, superiorly placed lag screw and longer tip apex distance.

To decrease the chances of lag screw cut out we recommend that fracture should be reduce accurately, superior screw placement of lag screw should be avoided and tip apex distance(TAD) should not exceed $25 \mathrm{~mm}$.

Copyright@ 15 Sep, 2017.

\section{REFERENCES}

1. Yang $Y H$, Wang YR, Jiang SD, Jiang LS. Proximal femoral nail anti rotation and third-generation Gamma nail: which is a better device for the treatment of intertrochanteric fractures? Singapore Med J. 2013; 54:446-450.

2. Matre K, Vinje T, Havelin LI, Gjertsen JE, Furnes O, Espehaug $B$, et al. Trigenintertan intramedullary nail versus sliding hip screw: A prospective, randomized multicenter study on pain, function, and complications in 684 patients with an inter- trochanteric or subtrochanteric fracture and one year of follow-up. J Bone Joint Surg Am. 2013; 95(3):200208.

3. Kumar N, Kataria H, Yadav CS, Gadagoli BS, Raj $R$. Evaluation of proximal femoral locking plate in unstable extracapsular proximal femoral fractures: Surgical technique and midterm follow up results. Journal of clinical orthopaedics and trauma. 2014:137-45.

4. Schipper IB, Marti RK, van der Werken C. Unstable pertrochanteric femoral fractures: Extramedullary or intramedullary fixation. Review of literature. Injury. 2004; 35:142-151.

5. Verettas DA, Ifantidis P, Chatzipapas CN, Drosos Gl, Xarchas KC, Chloropoulou $P$ et al. Systematic effects of surgical treatment of hip fractures: Gliding screwplating vs intramedullary nailing. Injury $2010 ; 41: 279$ 84.

6. Sedighi A, Sales JG, Alvi S. The prognostic value of tip-to-apex distance (TAD index) in intertrochanteric fractures fixed by dynamic hip screw. Orthop Rev (Pavia) 2012; 4(4):32-36.

7. Baumgaertner MR, Curtin SL, Lindskog DM, Keggi $\mathrm{JM}$. The value of the tip-apex distance in predicting failure of fixation of peritrochanteric fractures of the hip. J Bone Joint Surg Am 1995; 77(7):1058-64.

8. Lindskog D.M., Baumgaertner M.R. Unstable intertrochanteric hip fractures in the elderly. $\mathrm{J} \mathrm{Am}$ AcadOrthopSurg 2004; 12:179-190.

9. Pervez H., Parker M.J., Vowler S. Prediction of fixation after sliding hip screw fixation Injury 2004; 3:994-998.

10. Walton, NP, Wynn-Jones $H$, Ward MS, Wimhurst JA. Femoral neck-shaft angle in extra-capsular proximal femoral fracture fixation; does it make a TAD of difference? Injury 2005; 36(11):1361-4.

11. Marsh JL, Slongo TF, Agel J, Broderick JS, Creevey W, DeCoster TA et al. Fracture and dislocation classification compendium - 2007: orthopaedic trauma association classification, database and outcomes committee. J Orthop Trauma 2007; 21(10 Suppl):S1-133.

12. Cleveland M, Bosworth DM, Thompson FR, Wilson HJ, Ishizuka T. A ten-year analysis of intertrochanteric fractures of the femur. J Bone Joint Surg1959; 41:13991408.

13. Kyle RF, Gustilo RB, Premer RF. Analysis of six hundred and twenty-two intertrochanteric hip fractures. A retrospective and prospective study. J Bone Joint Surg Am 1976; 61:216-221. 
14. Parker MJ. Cutting-out of the dynamic hip screw related to its position. J Bone Joint Surg Br. 1992; 74(4):625.

15. Guven M, Yavuz U, Kadioglu B, Akman B, Kilincoglu $\mathrm{V}$, Unay $\mathrm{K}$ et al. Importance of screwposition in intertrochanteric fractures treated by dynamic hip screw. Orthop TraumatolSurg Res 2010; 96(1):21-7.

16. Hsueh KK, Fang CK, Chen CM, Su YP, Wu HF, Chiu FY. Risk factors in cutout of sliding hip screw in intertrochanteric fractures: An evaluation of 937 patients. Int Orthop2010; 34:1273-6.

17. Agni N, Sellers E, Johnson R, Gray A. Tip apex distance -Is it enough to predict implant failure? The Internet Journal of Orthopedic Surgery 2012; 19(3):1-5.

18. Andruszkow H, Frink M, Fromke C, Matityahu A, Zeckey C, Mommsen P, et al. Tip apex distance, hip screw placement, and neck shaft angle as potential risk factors for cut-out failure of hip screws after surgical treatment of intertrochanteric fractures. IntOrthop. 2012; 36(11): 2347-2354.
19. Bruijn KD, Hartog DD, Tuinbreijer W, Roukema G. Reliability of predictors for screw cutout in intertrochanteric hip fractures. J Bone Joint Surg Am. 2012; 94:1266-72.

20. Imaduddin S, Asim T, Razzak R. The value of the tipapex distance (TAD) in predicting failure of fi xation of Intertrochanteric fractures of femur. Pak J Surg $2014 ; 30(4): 320-326$.

21. Goh KL, Zamzuri Z, Mohd Arif S, Mohamed Azril MA. Intertrochanteric fracture fixation with Dynamic Hip Screw: Is tip-apex distance measurement useful for predicting fixation failure? IMJM 2016; 15(1):31-34.

22. Johnson LJ, Cope MR, Shahrokhi S, Tamblyn P. Measuring tip-apex distance using a picture archiving and communication system (PACS). Injury, 2008; 39(7): 786-90.

23. Mohan R, Karthikeyan R, Sonanis SV. Dynamic hip screw: Does side make a difference? Effects of clockwise torque on right and left DHS. Injury 2000; 31: 697-9.

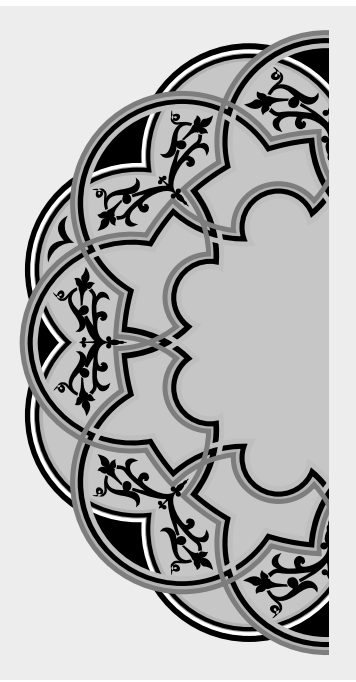

\title{
"To wish you were someone else is to waste the person you are."
}

\author{
Unknown
}

\section{AUTHORSHIP AND CONTRIBUTION DECLARATION}

\begin{tabular}{|c|c|c|c|}
\hline Sr. \# & Author-s Full Name & Contribution to the paper & Author $=$ s Signature \\
\hline 1 & Faaiz Ali Shah & $\begin{array}{l}\text { Conception \& design, Data } \\
\text { collection. }\end{array}$ & \\
\hline 2 & Waqar Alam & Data interpretation, Drafting & \\
\hline 3 & Main Amjad Ali & Final approval of study & \\
\hline
\end{tabular}

